\title{
Spontaneous Closure of the Macular Hole in a Patient with Acquired Vitelliform Lesion
}

\author{
Masanori Fukumoto Shou Oosuka Takaki Sato Teruyo Kida \\ Tsunehiko Ikeda \\ Department of Ophthalmology, Osaka Medical College, Takatsuki, Japan
}

\section{Keywords}

Macular hole · Acquired vitelliform lesion - Vitrectomy · Atopic retinal detachment

\begin{abstract}
In this paper, we report an extremely rare case of spontaneous closure of a macular hole $(\mathrm{MH})$ that developed in a patient in whom acquired vitelliform lesion (AVL) occurred after vitrectomy for atopic retinal detachment (ARD). A 32-year-old male developed ARD in both eyes, and retinal reattachment was achieved after vitrectomy. Five years after surgery, optical coherence tomography showed localized serous retinal detachment (SRD) and a granular lesion with a higher brightness in the subretinal fluid, thus leading to the diagnosis of AVL. One month later, an $\mathrm{MH}$ developed, and a follow-up examination performed 6 weeks later revealed that the $\mathrm{MH}$ had spontaneously closed and the SRD decreased. In the fovea, fluorescein angiography revealed a window defect due to atrophy of the retinal pigment epithelium (RPE). These findings in this present case suggest the possibility that RPE dysfunction was involved in the development of $\mathrm{AVL}$ and $\mathrm{MH}$.




\section{Introduction}

The disease concept of acquired vitelliform lesion (AVL) was first proposed by Freund et al. [1] in 2011. In that study, the authors reported that the disease is characterized by fundus findings that are similar to those of unilateral adult-onset vitelliform foveomacular dystrophy (AVFD), and made a distinction between AVFD, which is considered hereditary, and AVL, which occurs due to other causes.

Here, we report an extremely rare case of a macular hole (MH) that spontaneously closed during the clinical course in a patient in whom MH-complicated AVL occurred after vitrectomy for atopic retinal detachment (ARD).

\section{Case Report}

This study involved a 32-year-old male patient with systemic atopic dermatitis. His family history was unremarkable. ARD developed in his left eye in 2003 and in his right eye in 2012, yet in both eyes, vitrectomy surgery resulted in the retinas being successfully reattached and the clinical course was favorable. After surgery, a corrected visual acuity (VA) of $1.0 \mathrm{OD}$ and 0.6 OS was maintained. Fundus findings of the patient's right eye revealed a somewhat yellowish lesion at the inferior area of the fovea (Fig. 1). On September 10, 2017, the patient once again presented at our department's outpatient clinic with a primary complaint of decreased central vision in his right eye. Ophthalmoscopic examination of the right eye showed yellow exudates in the fovea of the retina (Fig. 2a). OCT findings revealed localized serous retinal detachment and a granular lesion with a higher brightness in the subretinal fluid (Fig. 2b). One month later, an MH developed (Fig. 3a), and a granular shadow with a higher brightness was observed at the base of the MH (Fig. 3b). Upon examination, the corrected VA in the patient's right eye was 0.6. Fluorescein angiography findings revealed hyperfluorescence due to a window defect (Fig. 4a), which corresponded with the shape of yellowish lesion in Figure 1. Indocyanine green angiography showed no choroidal neovascularization (Fig. 4b). The patient was then followed up, with vitrectomy being considered as an option, yet a follow-up examination performed 6 weeks later revealed that the MH had spontaneously closed and that the subretinal exudative changes had decreased (Fig. 5a). Moreover, the serous retinal detachment gradually disappeared (Fig. 5b). One month later, the corrected VA in his right eye improved to 0.8 .

\section{Discussion}

The disease concept of AVL was established relatively recently, and the number of reported cases remains small [1-9]. AVL is usually unilateral, and the most common symptoms are scotoma and metamorphopsia. The visual impairment is usually mild; however, it is greater in patients with a large vitelliform lesion with a thickened retina and those with choroidal neovascularization. In cases of AVL, the fundus findings and OCT findings are remarkably similar to those of AVFD. In regard to the pathogenesis of AVL, it has been theorized that dysfunction of phagocytosis of the photoreceptor outer segments due to dysfunctional retinal pigment epithelium, as in AVFD, leads to the formation of yellow lesions originating from the remnants of the photoreceptor outer segments and macrophages recruited to ingest them. In the present case, fluorescein angiography findings revealed a window defect in the macular 
area of the patient's right eye, thus suggesting the possibility that the retinal pigment epithelium in the macular area might have been damaged by ARD.

Chen et al. [2] reported that intraretinal hyperreflective foci, typically observed in patients with diabetic macular edema, were observed by OCT in $9.8 \%$ of patients with AVL. In that study, they reported that pigment cells had accumulated in the external granular layer at the location where intraretinal hyperreflective foci were identified on autopsy. Lima et al. [3] reported that the strong fundus autofluorescence seen in AVL was attributable to large drusen between the retinal pigment epithelium and Bruch's membrane. Bamonte et al. [4] reported a case of reabsorption of AVL after vitrectomy, thus suggesting that retinal vitreous traction may be involved in the pathogenesis of the disease.

There are several reported cases of AVFD complicated by MH. Noble and Chang [10] reported that thinning of the retina, along with regression of the yellow lesion, led to the formation of the MH. Soliman [11] reported cases of AVFD complicated by MH in which retinal detachment progressed around the lesion. Arnold et al. [12] examined the lesions with OCT, and reported that the disappearance of photoreceptor cells in the fovea prior to the onset of $\mathrm{MH}$ and subsequent thinning of the inner layer of the retina led to the formation of the $\mathrm{MH}$. Goldberg and Freund [13] suggested a similar pathogenic mechanism; however, considering the presence of $\mathrm{MH}$ in patients without epiretinal membrane or retinal-vitreous body adhesions, they reported that retinal vitreous traction was less involved in the development of $\mathrm{MH}$.

To the best of our knowledge, the study by de Souza et al. [14] was the only one to report a case in which AVFD complicated by MH was aggressively treated with vitrectomy. In that study, they reported that inner limiting membrane peeling and gas tamponade were performed after vitrectomy, as in cases of ordinary idiopathic $\mathrm{MH}$, yet the patient had recurrence and underwent repeat surgery using silicone oil with a specific gravity greater than one, with favorable results and successful removal of the silicone oil. However, no studies have reported spontaneous closure of $\mathrm{MH}$, as in the present case. Furthermore, it should be noted that the study by Goldberg and Freund [13] was the only previous study reporting a case of AVL associated with MH.

As seen in the OCT findings in this present case, an MH associated with AVFD or AVL is different from an ordinary idiopathic $\mathrm{MH}$, and is frequently associated with a granular lesion with a higher brightness at the base of the $\mathrm{MH}$. In this present case, it remains unclear as to how the behavior of the substance played a role in the spontaneous closure of the MH. One possible explanation is that the substance acted as scaffolding to promote the regeneration of glial cells, thereby leading to the spontaneous closure of the MH. When seeing patients in whom MH occurs in association with AVFD or AVL, close follow-up without aggressive treatment for a certain period of time can be a therapeutic option when considering the possibility that spontaneous closure of the MH may occur.

\section{Acknowledgments}

The authors wish to thank John Bush for editing the manuscript. 


\section{Statement of Ethics}

The protocols of this study were approved by the Ethics Committee of Osaka Medical College, Takatsuki, Osaka, Japan. In accordance with the tenets set forth in the Declaration of Helsinki, prior written informed consent was obtained from the patient.

\section{Disclosure Statement}

There are no conflicts of interest to report for all authors.

\section{Author Contributions}

M.F. and T.S.: equal contribution of patient management, conception of the paper, data analysis and interpretation, manuscript drafting, and literature search. S.O. and T.K.: co-writing of the manuscript and literature search. T.I.: design of the paper, co-writing of the manuscript, literature search, manuscript editing and revision, as well as final approval. All authors read and approved the final manuscript.

\section{References}

1 Freund KB, Laud K, Lima LH, Spaide RF, Zweifel S, Yannuzzi LA. Acquired Vitelliform Lesions: correlation of clinical findings and multiple imaging analyses. Retina. 2011 Jan;31(1):13-25.

2 Chen KC, Jung JJ, Curcio CA, Balaratnasingam C, Gallego-Pinazo R, Dolz-Marco R, et al. Intraretinal hyperreflective foci in acquired vitelliform lesions of the macula: clinical and histologic study. Am J Ophthalmol. 2016 Apr;164:89-98.

3 Lima LH, Laud K, Freund KB, Yannuzzi LA, Spaide RF. Acquired vitelliform lesion associated with large drusen. Retina. 2012 Apr;32(4):647-51.

4 Bamonte G, Appeltans A, Cicinelli MV, Querques G. Reabsorption of acquired vitelliform lesions in vitreomacular disorders after vitrectomy. Retin Cases Brief Rep. 2020 Winter;14(1):10-14.

5 Chae B, Dhrami-Gavazi E, Dansingani KK, Freund KB, Lee W, Yannuzzi LA. Multimodal imaging of combined hamartoma of the retina and retinal pigment epithelium associated with an acquired vitelliform lesion. Int J Retina Vitreous. 2015 Dec;1(1):23.

6 Rocha Bastos R, Ferreira CS, Brandão E, Falcão-Reis F, Carneiro ÂM. Multimodal image analysis in acquired vitelliform lesions and adult-onset foveomacular vitelliform dystrophy. J Ophthalmol. 2016;2016:6037537.

7 Corvi F, Querques G. Progressive development of acquired vitelliform lesion secondary to chorioretinal folds. J Fr Ophtalmol. 2015 Nov;38(9):898-9.

8 Curcio CA, Balaratnasingam C, Messinger JD, Yannuzzi LA, Freund KB. Correlation of type 1 neovascularization associated with acquired vitelliform lesion in the setting of age-related macular degeneration. Am J Ophthalmol. 2015 Nov;160(5):1024-1033.e3.

9 Gonçalves NM, Carneiro AM, Brandão E, Falcão-Reis FM. Multimodal imaging of acquired vitelliform lesion diagnosed at pseudohypopyon stage. Case Rep Ophthalmol Med. 2013;2013:461758.

10 Noble KG, Chang S. Adult vitelliform macular degeneration progressing to full-thickness macular hole. Arch Ophthalmol. 1991 Mar;109(3):325.

11 Soliman MM. Vitelliform macular dystrophy: a cause of macular holes with retinal detachments. Eye (Lond). 1994;8(Pt 4):484-7.

12 Arnold JJ, Sarks JP, Killingsworth MC, Kettle EK, Sarks SH. Adult vitelliform macular degeneration: a clinicopathological study. Eye (Lond). 2003 Aug;17(6):717-26.

13 Goldberg N, Freund KB. Progression of an acquired vitelliform lesion to a full-thickness macular hole documented by eye-tracked spectral-domain optical coherence tomography. Arch Ophthalmol. 2012 Sep;130(9):1221-3.

14 de Souza CF, Polkinghorne PJ, Riley AF. Heavy silicone oil effective in macular hole surgery associated with adult vitelliform macular dystrophy. Clin Exp Ophthalmol. 2012 Jan-Feb;40(1):e111-2. 


\section{Case Reports in Ophthalmology}

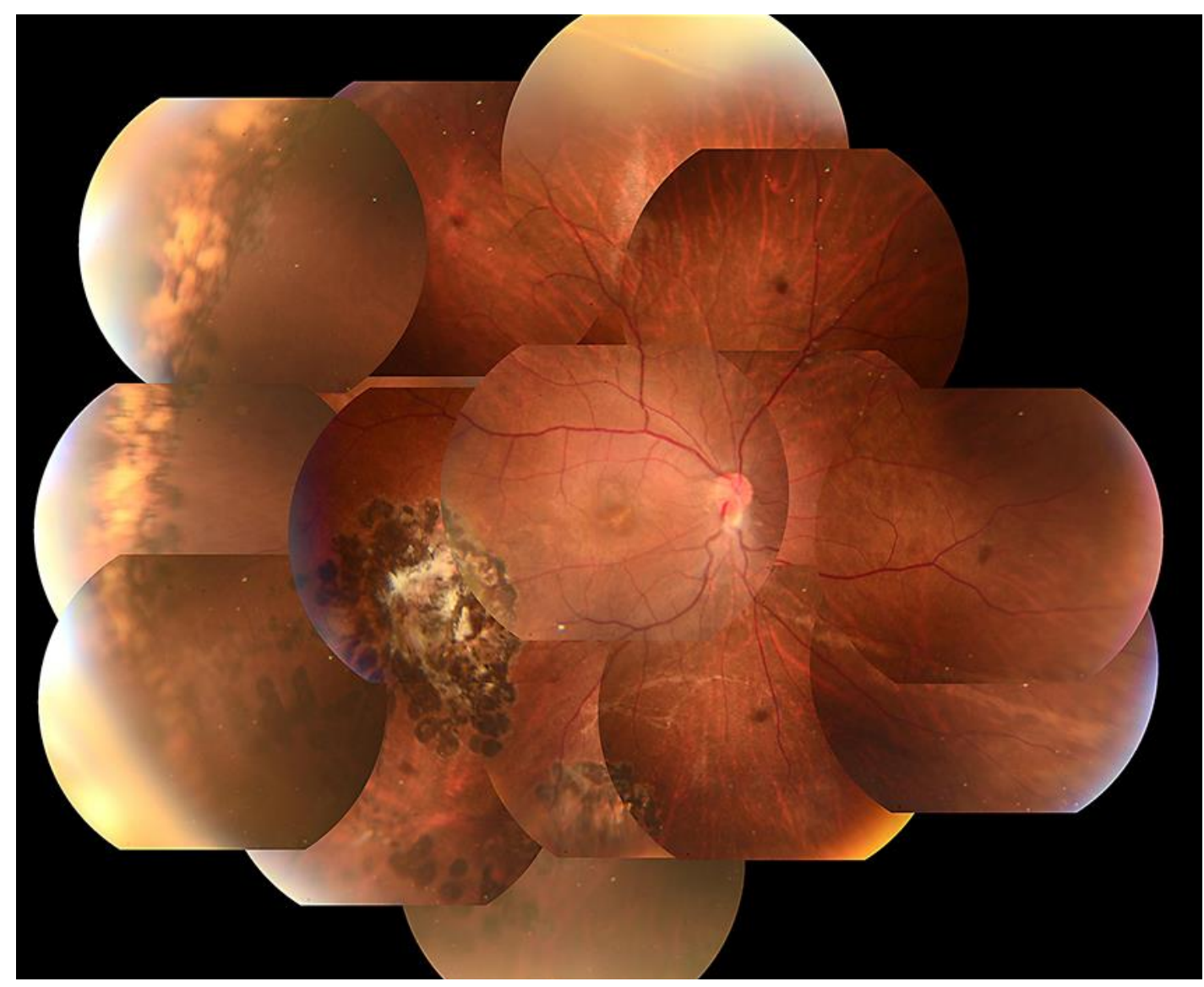

Fig. 1. Fundus photographs of the patient's right eye obtained after vitreous surgery for atopic retinal detachment. Retinal reattachment was achieved in both eyes after vitrectomy, and mild retinal pigment epithelial damage was observed in the fovea.
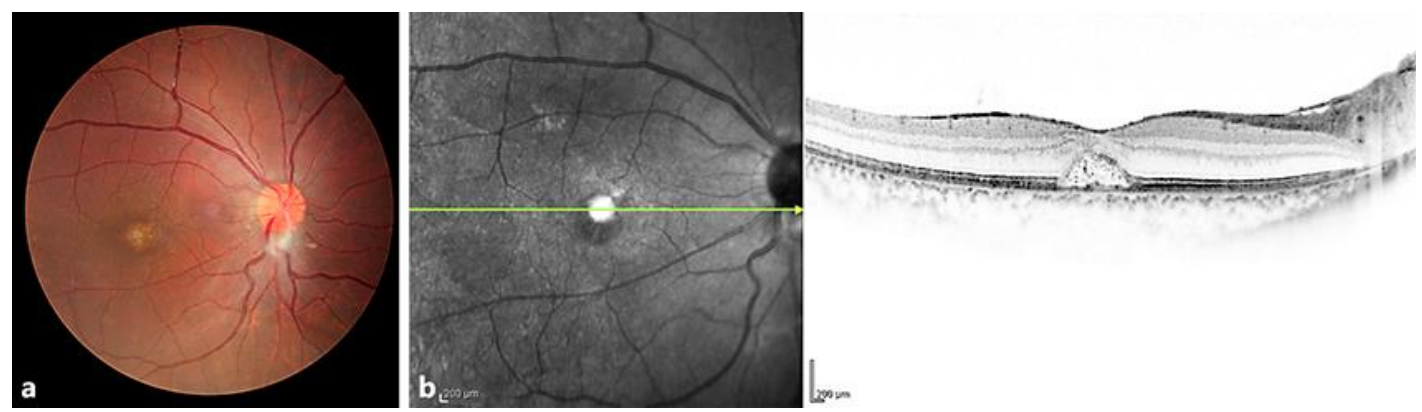

Fig. 2. Fundus photograph and OCT imaging of the patient's right eye on September 10, 2017, showing yellow exudates in the fovea of the retina (a) and localized serous retinal detachment and granular lesion with a higher brightness in the subretinal fluid (b). 


\section{Case Reports in Ophthalmology}

www.karger.com/cop

Fukumoto et al.: Spontaneous Closure of the Macular Hole in a Patient with AVL
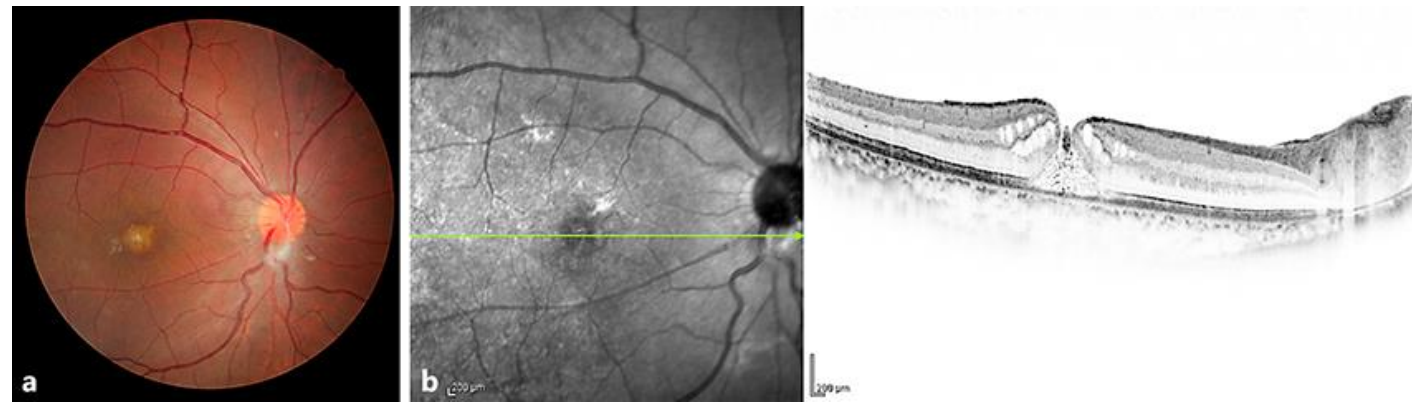

Fig. 3. Fundus photograph and OCT imaging of the patient's right eye on October 9th, 2017. MH occurred (a), and a granular shadow with a higher brightness was observed at the base of the MH (b).
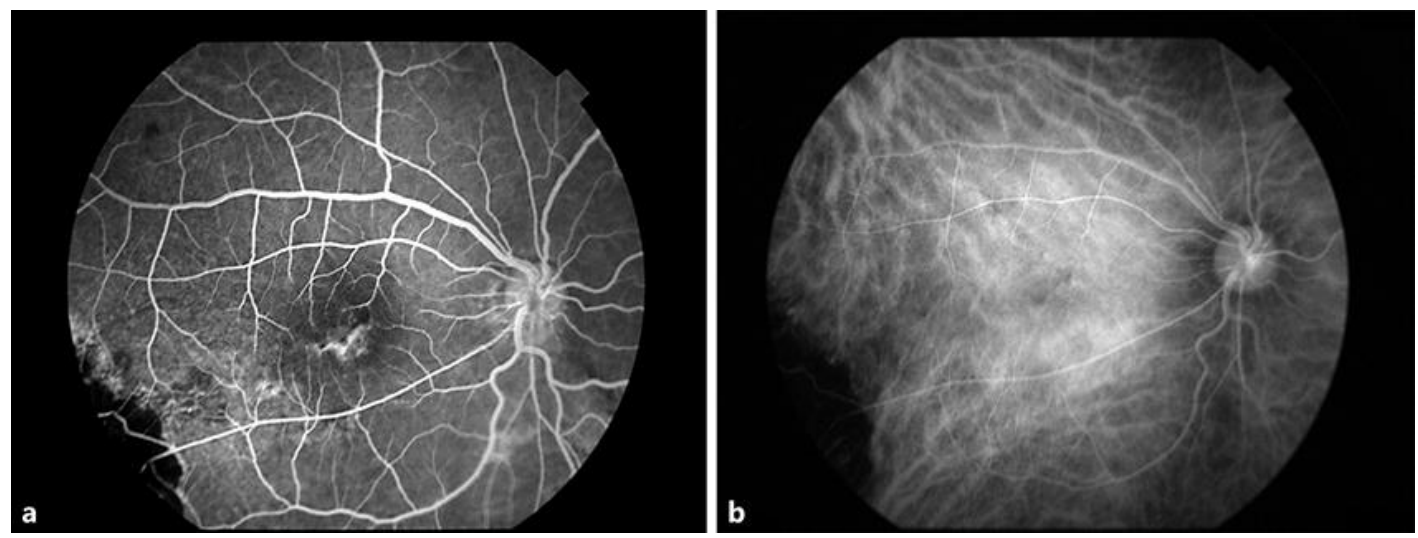

Fig. 4. Fluorescein angiography (FA) and indocyanine green angiography (IA) images of the patient's right eye obtained on October 9th, 2017. FA showed hyperfluorescence due to a window defect (a), and IA showed no choroidal neovascularization (b).
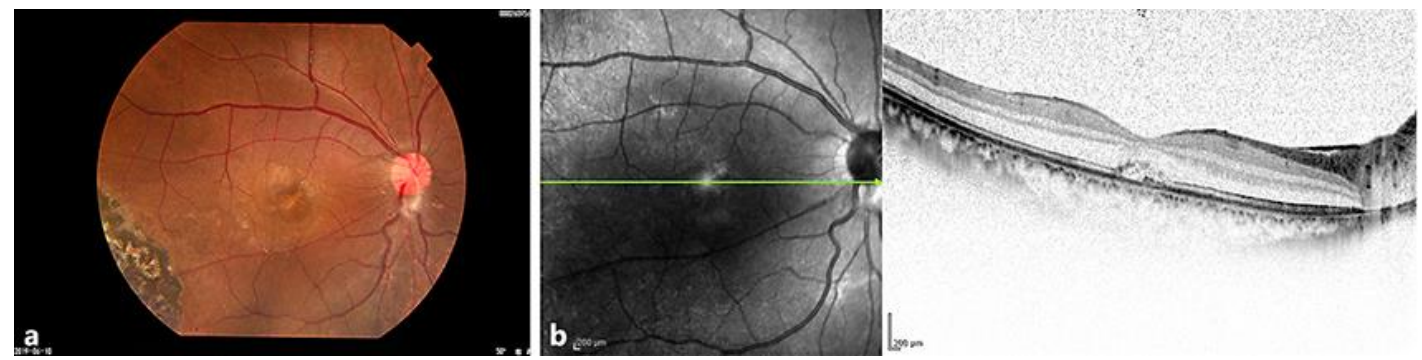

Fig. 5. Fundus photograph and OCT imaging of the patient's right eye obtained on November 23rd, 2017. The MH spontaneously closed and the subretinal exudative changes decreased (a), and the serous retinal detachment gradually disappeared (b). 